\title{
A Morphological Optical Survey of Nearby AGN
}

\author{
C.S. Boschetti
}

\author{
Dipartimento di Astronomia, Università di Padova, Italy
}
S. Ciroi ${ }^{1)}$, J. Funes ${ }^{2)}$, A. Omizzolo ${ }^{2)}$, P. Rafanelli ${ }^{1)}$, G.M. Richter ${ }^{3)}$, A. Rifatto $^{4)}, \mathrm{J}$. Vennik ${ }^{5)}$

1) Dipartimento di Astronomia, Università di Padova, Italy; ${ }^{2)}$ Specola Vaticana, Città del Vaticano, ${ }^{3}$ Astrophysikalisches Institut Potsdam, Germany; ${ }^{4)}$ Osservatorio Astronomico di Capodimonte, Napoli, Italy;

5) Tartu Observatory, Toravere, Estonia

\begin{abstract}
We present preliminary results of an optical survey of a spectroscopically selected sample of nearby AGNs $(z<0.1)$. The aim of the program that we are currently carrying out is to demonstrate that the activity phenomenon is strongly related to galaxy interactions. In detail, we want to study the morphology of our sample of galaxies in order to reveal the presence of features like bars, rings, double nuclei, isophotal distortions and tidal tails, most of which are not detectable on the POSS plates. The work is based on the analysis of broad band $B, V$ and $R$ images, the study of their photometric parameters and the results of the application of a Laplacian adaptive filter, able to enhance hidden and faint structures.
\end{abstract}

\section{Introduction}

Seyfert galaxies are the nearest, and hence most widely studied AGNs, usually associated with spirals, but some are also found in ellipticals.

Whether Seyferts tend to be interacting with nearby companions (Dahari 1984) or not (Bushouse 1986) is still a matter of debate. Nevertheless, several statistical studies carried out within our group have shown an excess of Seyferts among galaxy pairs and an excess of physical companions in samples of Seyferts (Rafanelli et al. 1995, 1997; Salvato \& Rafanelli 1997).

Other clues to the connection between interaction and activity come from morphological studies, which indicate that many Seyferts are amorphous or disturbed galaxies (MacKenty 1990), and from N-body simulations, which show how disk instabilities in colliding galaxies can lead rapidly to the formation of strong bars and to inflows of gas fueling starburst and/or AGN activity before galaxy merging (Mihos \& Hernquist 1996; Barnes \& Hernquist 1996; Mihos 1999).

We present preliminary results of an optical imaging survey of a spectroscopically selected sample of nearby AGN $(\mathrm{z}<0.1)$. Only isolated galaxies are 
extracted, following the criteria given by Rafanelli et al. (1995), and studied to reveal morphological signatures of recent interactions like bars, double or multiple nuclei, isophotal distortions and tidal tails, most of which are not detectable on the POSS plates.

\section{Observations \& Data Analysis}

As examples of the AGN survey we are still currently carrying out, we present three galaxies: HE 1338-1423, ESO 362-G018 and NGC 2768. The first two objects were observed in April, 1995, at the ESO 2.2m telescope with EFOSC2 and with a typical seeing of $\sim 1^{\prime \prime}$. The $1 \mathrm{k} \times 1 \mathrm{k}$ CCD $(19 \mu$ pixel $)$ covered a field of $5.7^{\prime}$ with a scale of $0.336^{\prime \prime} / \mathrm{px}$. The data of the third object were taken in October, 1998 , at the VATT $1.8 \mathrm{~m}$ telescope, with a $2 \mathrm{k} \times 2 \mathrm{k}$ CCD camera $(15 \mu$ pixel) covering a field of $6.4^{\prime}$ with a a scale of $0.4^{\prime \prime} / \mathrm{px}$ (after binning). The seeing was $\sim 2^{\prime \prime}$.

Broad band $B, V$ and $R$ images were obtained for each galaxy and reduced within the IRAF environment then an Adaptive Filter procedure (Richter et al. 1991; Lorenz et al. 1993) was applied to strongly reduce the noise (smooth) and/or enhance hidden and faint structures (Laplacian).

The morphology of the galaxies was analyzed by fitting their isophotes with free ellipses (Jedrzejewski 1997) and plotting as a function of the equivalent radius $\left(R_{e q}\right)$ the brightness profile, the ellipticity $(e)$, position angle $(P . A$.$) and$ center $(x, y)$ of the ellipses, and the parameters which measure the deviation from perfect elliptical isophotes. In addition, a model of each object was built on the basis of the best-fitting ellipses and subtracted from the original image to study the residuals.

\section{Results}

HE 1338-1423 is a Seyfert galaxy $(\mathrm{z}=0.041)$ without any morphological classification. The $\mathrm{V}$ image and the isophotal analysis reveal a little bar with semimajor axis of $\sim 4^{\prime \prime}\left(\sim 3.2 \mathrm{kpc}\right.$, assuming $\left.\mathrm{H}_{0}=75 \mathrm{~km} \mathrm{~s}^{-1} \mathrm{Mpc}^{-1}\right)$ oriented at P.A. $\sim 160^{\circ}$. The brightness profile was fitted trying to take into account the contributions of the main components: a point source for the AGN, an exponential bulge, a bar and a disk. The galaxy is clearly disk dominated and the residuals of the fit reveal a large structure with at least three maxima at about $7^{\prime \prime}, 10^{\prime \prime}$ and $14^{\prime \prime}(\sim 5.6,8$ and $11.2 \mathrm{kpc})$, corresponding to different spiral arms. Such arms are very well enhanced by means of the Laplacian adaptive filter application.

ESO 362-G018 is a Seyfert $1.5(\mathrm{z}=0.012)$ classified in NED as S0/Sa. The $\mathrm{B}$ image shows an asymmetric structure, as indicated by the evident changing of the ellipses centers, a very bright and elliptical nucleus and an emission knot at $\sim 8^{\prime \prime}(\sim 1.8 \mathrm{kpc}) \mathrm{NE}$ of it. Two faint tidal tails are visible, one of $\sim 10^{\prime \prime}$ $(\sim 2.3 \mathrm{kpc})$ in the NW direction and the other very extended $(>30 \mathrm{kpc})$ to the South. The first one is clearly connected to the knot in the residual image, after the model subtraction, which shows also two other structures in the SW and SE directions. We suggest that the knot is in fact a companion galaxy in an advanced merging phase with the Seyfert. 
NGC 2768 is a LINER $(\mathrm{z}=0.004)$ galaxy classified as $\mathrm{S} 0$. The isophotal analysis of the $\mathrm{R}$ image confirms its early type and symmetric morphology: the brightness profile follows the $r^{1 / 4}$ De Vaucouleurs law typical of elliptical galaxies, the P.A. is almost constant around $\sim 95^{\circ}$ and the ellipticity increases smoothly outwards. Notwithstanding these properties, the 4 th order cosine coefficient $C_{4}$ (Bender et al. 1989) is negative up to $\sim 50^{\prime \prime}(\sim 4 \mathrm{kpc})$, indicating that the inner isophotes are boxy. As expected, the residual image shows missing light along the principal axes and excess light along two diagonal lines. This feature is believed to be the result of a merger remnant. (Hernquist \& Quinn 1989). These few examples extracted from the many data of our survey show that active galaxies with apparent regular morphology have systematically more complicated structures and hide very often traces of past or current interaction with other galaxies. In the future we plan to select and observe a control sample of non-active galaxies to put stronger constraints to the interaction-activity connection.

\section{References}

Barnes, J. \& Hernquist, L. 1996, ApJ, 471, 115

Bender, R., Surma, P., Döbereiner, S. et al. 1989, A\&A, 217, 35

Bushouse, H.A. 1986, AJ, 91, 255

Dahari, O. 1985, ApJS, 57, 643

Hernquist, L. \& Quinn, P.J. 1989, ApJ, 342, 1

Jedrzejewski, R.I 1987, MNRAS, 226, 747

MacKenty, J.W. 1990, ApJS, 72, 231

Mihos, C. \& Hernquist, L. 1996, ApJ, 464,641

Mihos, C 1999, preprint, astro-ph/9903115

Rafanelli, P., Violato, M. \& Baruffolo, A. 1995, AJ, 109, 1546

Rafanelli, P., Temporin, S. \& Baruffolo, A. 1997, AN, 318, 249

Salvato, M. \& Rafanelli, P. 1997, AN, 318, 237 